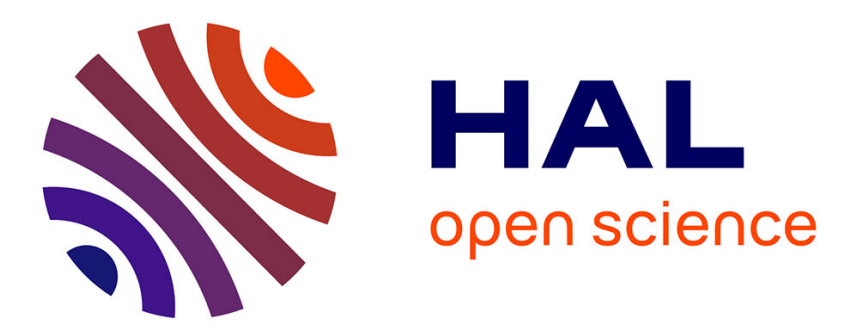

\title{
Equivalent source model from acousto-optic measurements and application to an acoustic pulse characterization
}

Pierre Lecomte, Quentin Leclere, Sébastien Ollivier

\section{To cite this version:}

Pierre Lecomte, Quentin Leclere, Sébastien Ollivier. Equivalent source model from acousto-optic measurements and application to an acoustic pulse characterization. Journal of Sound and Vibration, 2019, 450, pp.141-155. 10.1016/j.jsv.2019.03.012 . hal-02427018

\author{
HAL Id: hal-02427018 \\ https://hal.science/hal-02427018
}

Submitted on 22 Oct 2021

HAL is a multi-disciplinary open access archive for the deposit and dissemination of scientific research documents, whether they are published or not. The documents may come from teaching and research institutions in France or abroad, or from public or private research centers.
L'archive ouverte pluridisciplinaire HAL, est destinée au dépôt et à la diffusion de documents scientifiques de niveau recherche, publiés ou non, émanant des établissements d'enseignement et de recherche français ou étrangers, des laboratoires publics ou privés.

\section{(c) (1) $\$$}

Distributed under a Creative Commons Attribution - NonCommerciall 4.0 International 


\title{
Equivalent source model from acousto-optic measurements and application to an acoustic pulse characterization
}

\author{
Pierre Lecomte ${ }^{\mathrm{a}, *}$, Quentin Leclère ${ }^{\mathrm{a}}$, Sébastien Ollivier ${ }^{\mathrm{b}}$ \\ ${ }^{a}$ Univ Lyon, INSA-Lyon, LVA EA677, F-69621, Villeurbanne, France \\ ${ }^{b}$ Univ Lyon, Université Lyon 1, LMFA UMR CNRS 5509, Ecully, F-69134, France
}

\begin{abstract}
The paper describes an equivalent source model of acoustic sources from optical measurements using refracto-vibrometry. The acousto-optic effect is used to characterize the acoustic source in a non-invasive manner. The equivalent source model is constructed with a volumic distribution of monopole sources: its radiation should produce an acouto-optic effect which match with the measured one. The solution of this inverse problem gives the monopole strengths. This method is applied to the characterization of an acoustic pulse source. It is shown that the reconstructed pressure with the equivalent source matches the measured one within a $2 \mathrm{~dB}$ range. Moreover, the equivalent source dimensions, spatial location and directivity are in good accordance with expected characteristics of the real source.
\end{abstract}

Keywords: acousto-optic, inverse problem, refracto-vibrometry, acoustic pulse

\section{Introduction}

The characterization of acoustic sources is generally done with microphone arrays: from a set of discrete pressure measurements, the sources localization, their strengths or their radiation properties are characterized using multichannel signal processing. Popular approaches include beamforming [1], near field holography [2], equivalent source methods and inverse problems [3, 4], time reversal [5] or modal array signal processing [6].

\footnotetext{
${ }^{*}$ Corresponding author
} 
In an experimental context, a main drawback of the measurements with microphone arrays is the acoustic influence of the array itself, especially at high frequencies. When the wavelength is of the same order of magnitude as the microphones or their support, the diffraction compromise the measurement validity. For instance, the spark sources characterization with conventional microphone suffer from those problems at high frequencies [7].

It would be of great interest to have alternative acoustic sources characterization method, which do not suffer from diffraction effects and limited microphone response bandwidth. Especially, if the source characterization is precise enough, then it could be used as a calibration source [8]. This is the motivation of this work.

An approach of measuring sound in a non-invasive manner relies on the acousto-optic effect which describes the interaction of sound and light when a acoustic waves travels in a transparent medium. The resulting techniques are used in ultrasound acoustics $[9,10,11]$ and more recently in audible domain $[12,13]$.

The measurement of acousto-optic effect can be done with interferometric techniques $[12,14]$. However, by doing so, the optical measurement is proportional to the pressure spatial integration along the laser path. In order to solve this integral and find the acoustic pressure, hypothesis on the source radiation can be done. For instance, a radially symmetric radiation hypothesis allows to use the Abel transform [14]. Otherwise, the acoustooptic tomography allows the characterization of the sound pressure field in a plane [13]. Besides, for a qualitative characterization of the sound field, a Laser Doppler Vibrometer (LDV) can be used. In this case, the apparent output velocity given by the LDV is proportional to the acousto-optic effect. This latter technique is called refracto-vibrometry [15, 16, 17].

In this context, one proposes to characterize acoustic sources by solving and inverse problem based on optical measurement. The measurements are obtained from a LDV whose laser beam passes through the sound pressure field with several angles of incidence and is reflected on a rigid surface. An acousto-optic Green function for monopole is highlighted and used to describe the acoustic sources as a combination of monopoles sources, which strengths are found by solving a regularized inverse problem.

The paper is organized as follows: The acousto-optic effect is recalled in and detailed for a monopole source in Sec. 2 and Appendix C. In particular, the effects of finite duration measurements are studied in Appendix B. Section 3 describes the Equivalent Source Method (ESM) inverse problem 
from acousto-optic measurements. Then, an experimental implementation of the proposed method is done for a spark source through Secs. 4 and 5 . Particularly, the experimental method is described in Sec. 4 and the ESM and signal processing parameters are detailed in Sec. 5. Results are presented and discussed in Secs. 5.4 and 5.5. The paper concludes in Sec. 6.

\section{Acousto-optic effect}

This section recalls the acousto-optic effect which allows the measurement of sound with optical methods.

As an acoustic wave propagates in air, the variation of acoustic pressure induces a local variation of air refractive index. This phenomenon is called the acousto-optic effect and has been widely studied in ultrasound $[9,10,11]$ and more recently for audible frequencies range $[12,13,8]$. If the acoustic pressure $p$ is much smaller than the static pressure $p_{0}$, which is generally admitted in linear acoustics, the air refractive index can be approximated with the following linear equation:

$$
n \simeq n_{0}+\frac{n_{0}-1}{\gamma p_{0}} p,
$$

where $n$ is the air refractive index, $p$ is the acoustic pressure, $\gamma=1.4$ is the ratio of specific heats, $n_{0}=1.0002772$ and $p_{0}$ are the air refractive index and atmospheric pressure under static condition.

To measure the acousto-optic effect, an interferometer using a laser beam

is commonly used. As the latter travels through the sound pressure field, a phase shift is induced and is expressed by $[13,14]$ :

$$
\phi=k_{0} \int_{\mathbf{L}} n \mathrm{~d} l,
$$

where $k_{0}=\omega_{0} / n_{0}$ is the wave number of light in vacuum with $\omega_{0}$ the laser beam pulsation, and $\mathbf{L}$ is the path followed by the laser beam.

\subsection{Measuring the acousto-optic effect with a Laser Doppler Vibrometer}

A Laser Doppler Vibrometer (LDV) is a device used to measure the velocity of vibrating structures. A laser beam is emitted by the LDV, travels through a sound pressure field and reflects back on a vibrating surface. The 
LDV output is a temporal signal which is proportional to the time derivative of the laser beam phase. From Eqs. (2) and Eq. (1) one has:

$$
\frac{1}{k_{0} n_{0}} \frac{\mathrm{d} \phi}{\mathrm{d} t}=\frac{\mathrm{d} L_{0}}{\mathrm{~d} t}+\frac{\left(n_{0}-1\right)}{\gamma n_{0} p_{0}} \frac{\mathrm{d}}{\mathrm{d} t} \int_{\mathbf{L}} p(l, t) \mathrm{d} l,
$$

where $L_{0}$ is the laser beam length, that is to say the distance between the laser origin and the reflecting surface. The first term in the right-hand side of Eq. (3) is the velocity of the vibrating surface. The right term of the righthand side of Eq. (3) traduces the acousto-optic effect. In the case where the reflecting surface does not vibrate, $\mathrm{d} L_{0} / \mathrm{d} t \simeq 0$. Thus, the acousto-optic effect is dominant and the LDV apparent velocity is given by:

$$
v(t)=\frac{1}{k_{0} n_{0}} \frac{\mathrm{d} \phi}{\mathrm{d} t}=\frac{n_{0}-1}{\gamma p_{0} n_{0}} \frac{\mathrm{d}}{\mathrm{d} t} \int_{\mathbf{L}} p(l, t) \mathrm{d} l,
$$

In the frequency domain, the apparent velocity of the LDV is given by [12]:

$$
v(\omega)=\frac{n_{0}-1}{\gamma p_{0} n_{0}} \mathrm{i} \omega \mathscr{F}\left(\int_{\mathbf{L}} p(l, t) \mathrm{d} l\right),
$$

where $\mathscr{F}$ is the Fourier transform operator with temporal convention $e^{+\mathrm{i} \omega t}$, $\mathrm{i}=\sqrt{-1}$ is the imaginary unit. This indirect use of an LDV to measure sound is also referred as refracto-vibrometry $[15,16,17]$.

\subsection{Acousto-optic effect for a monopole source}

A monopole source with strength $q(t)$ is located at a point $\mathbf{x}_{s}$ in space. The pressure radiated at a point $\mathbf{x}$ is given by :

$$
p(\mathbf{x}, t)=\frac{q(t) * \delta\left(t-\frac{\left\|\mathbf{x}-\mathbf{x}_{s}\right\|}{c}\right)}{4 \pi\left\|\mathbf{x}-\mathbf{x}_{s}\right\|},
$$

where $c$ is the sound speed in air, $\delta$ is the delta Dirac distribution and $*$ is the convolution operator.

Inserting Eq. (6) in Eq. (4) one has:

$$
\frac{1}{k_{0} n_{0}} \frac{\mathrm{d} \phi}{\mathrm{d} t}=\frac{\mathrm{d} q(t)}{\mathrm{d} t} \frac{\left(n_{0}-1\right)}{4 \pi \gamma p_{0} n_{0}} * \underbrace{\int_{\mathbf{L}} \frac{\delta\left(t-\frac{\left\|\mathbf{x}-\mathbf{x}_{s}\right\|}{c}\right)}{\left\|\mathbf{x}-\mathbf{x}_{s}\right\|} \mathrm{d} l}_{I(t)},
$$


One considers the integral term in Eq. (7), denoted $I(t)$. A laser beam emitted by the LDV is crossing the sound pressure field at a distance $a$ from $\mathbf{x}_{s}$. From here, for sake of simplicity, one works in the plane containing the point $\mathbf{x}_{s}$ and the laser beam. In this plane, the origin $O$ is chosen at point $\mathbf{x}_{s}$. The local coordinate system is defined with unit vectors $\mathbf{u}=\left[\begin{array}{ll}1 & 0\end{array}\right]^{T}$ and $\mathbf{v}=\left[\begin{array}{ll}0 & 1\end{array}\right]^{T}$ such as $\mathbf{u}$ is parallel at the laser beam. The situation is displayed in Fig. 1: the acoustic pressure is null everywhere except at the radius $r=c t$. The distance between the LDV and the rigid reflector is denoted $L$. If one

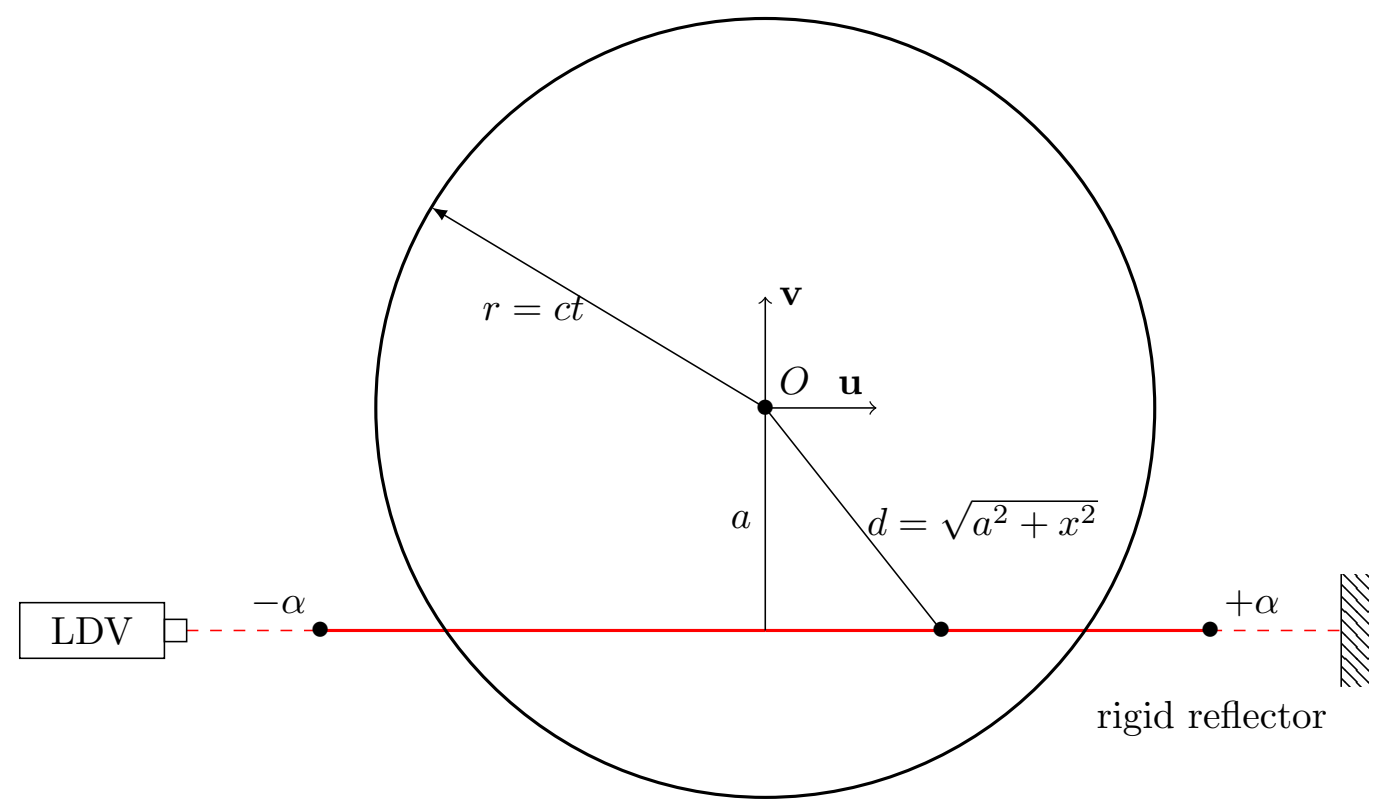

Figure 1: Geometrical layout for the measurement of the acousto-optic effect induced by a Dirac pulse.

restrains the signal acquisition duration for the LDV from time $t=0$ to time $t=\sqrt{a^{2}+\alpha^{2}} / c$, the Dirac pulse travels up to the distance $r=\sqrt{\alpha^{2}+a^{2}}$ from origin. Therefore, the integration path $\mathbf{L}$ for the term $I(t)$ in Eq. (7) can be reduced to the interval $\left[\begin{array}{ll}-\alpha & \alpha\end{array}\right]$ :

$$
I(t)=\int_{-\alpha}^{\alpha} \frac{\delta\left(t-\frac{\sqrt{a^{2}+x^{2}}}{c}\right)}{\sqrt{a^{2}+x^{2}}} \mathrm{~d} x
$$

The computation of $I(t)$ in Eq. 8 is detailed in Appendix A. In the frequency domain, $I(t)$ can be approximated by a Bessel function of second kind and 
degree $0, K_{0}$ :

$$
I(\omega) \simeq 2 K_{0}(\mathrm{i} \omega a / c)
$$

Note that this approximation is exact in the case of an infinitely long laser beam, see Appendix A. A study of the approximation error is conducted in Appendix B. Finally, by inserting Eqs. (9) and (6) into Eq. (5), the acousto-optic effect for a monopole gives the LDV apparent velocity approximated by:

$$
v(\omega, a)=\frac{\mathrm{i} \omega q(\omega)\left(n_{0}-1\right)}{4 \pi \gamma p_{0} n_{0}} 2 K_{0}(\mathrm{i} \omega a / c) .
$$

\section{Equivalent sources method from acousto-optic measurements}

The equivalent source method (ESM) seeks to find a distribution of simple sources modeling the sound pressure field radiated by the acoustic source to be characterized $[3,18,19]$. Usually based on microphone measurements, the ESM is adapted in this work for acousto-optic measurements using developments proposed in the Sec. 2. From Eq. 10, a distribution of $N$ monopoles sources produces an apparent velocity at point $\mathbf{x}_{m}$ given in the frequency domain by:

$$
\hat{v}_{m}=\sum_{n=1}^{N} q_{n} G\left(\mathbf{x}_{m}, \mathbf{x}_{n}\right),
$$

where, $q_{n}$ and $\mathbf{x}_{n}$ are respectively the strength and the location of the $n$-th monopole. $G\left(\mathbf{x}_{m}, \mathbf{x}_{n}\right)$ is the acousto-optic Green function given by:

$$
G\left(\mathbf{x}_{m}, \mathbf{x}_{n}\right)=\frac{\mathrm{i} \omega\left(n_{0}-1\right)}{4 \pi \gamma p_{0} n_{0}} 2 K_{0}\left(\frac{\mathrm{i} \omega}{c} a\left(\mathbf{x}_{m}, \mathbf{x}_{n}\right)\right) .
$$

The function $a\left(\mathbf{x}_{m}, \mathbf{x}_{n}\right)$ in Eq. (12) is the normal distance from the $n$-th monopole position $\mathbf{x}_{n}$ to a laser beam originating from a LDV and passing by point $\mathbf{x}_{m}$. The analytical expression of $a\left(\mathbf{x}_{m}, \mathbf{x}_{n}\right)$ is given in Appendix $\mathrm{C}$. A matrix formulation for $M$ spatial locations is given by:

$$
\hat{\mathbf{v}}=\mathbf{G q},
$$

where $\mathbf{v}^{(\hat{M} \times 1)}$ is the apparent velocity vector, $\mathbf{q}^{(N \times 1)}$ is the source strengths vector and $\mathbf{G}^{(M \times N)}$ is the matrix of acousto-optic Green functions.

The apparent velocity vector for the acoustic source to be modeled is denoted $\mathbf{v}^{(M \times 1)}$. This vector is measured with a LDV at $M$ spatial locations. 
The complex error vector $\mathbf{e}^{(M \times 1)}$ between the $\mathbf{v}$ and $\hat{\mathbf{v}}$ is defined as:

$$
\mathbf{e}=\mathbf{v}-\hat{\mathbf{v}}
$$

In order to find the source strengths $\mathbf{q}$ which minimize $\|\mathbf{e}\|^{2}$ in Eq. (14), while remaining a constrained energy solution, one defines the following regularized cost function $J[20]$ :

$$
J=\|\mathbf{v}-\mathbf{G q}\|^{2}+\lambda^{2}\|\mathbf{W q}\|^{2}
$$

where $\|\cdot\|$ denotes the 2-norm, $\lambda$ is a Tikhonov regularization parameter [21], and $\mathbf{W}^{(N \times N)}$ is a diagonal weighting matrix. In the reported cases, the choice of $\lambda$ is done in a Bayesian framework [22]. The solution q minimizing the cost function $J$, is given by [20]:

$$
\mathbf{q}=\left(\mathbf{G}^{H} \mathbf{G}+\lambda^{2} \mathbf{W}^{H} \mathbf{W}\right)^{-1} \mathbf{G}^{H} \mathbf{v},
$$

where ${ }^{H}$ is the Hermitian transpose conjugate operator.

\section{Experimental Method}

From this section, an experimental implementation of the method described in Sec. 3 is conducted for an acoustic pulse.

\subsection{Apparatus}

The experimental layout is shown in Fig. 2. A top view scheme is shown in Fig. 3. The acoustic pulse is generated with an electric spark between two metal electrodes spaced $10 \mathrm{~mm}$ apart. The electrodes diameter is $3 \mathrm{~mm}$. The electrical circuit comes from a piezoelectric oven spark ignitor. The structure supporting the electrodes is built with $2-\mathrm{mm}$ and $3-\mathrm{mm}$ thin metal rods, in order to limit its acoustical influence at high frequencies. It can rotate around the vertical axis. This allows measurements with several source orientations $\theta$, as shown in Fig. 3. The laser beam is emitted by a Polytech PSV 400(C LDV as shown on the left of Fig. 2. It crosses the sound pressure field and reflects back on the rigid reflector. 


\subsection{Geometrical layout}

The origin $O$ of the Cartesian coordinate system is chosen in the middle of the two electrodes. The $x$-axis is pointing towards the rigid reflector, the $z$-axis toward the ceiling and the $y$-axis is such that the coordinate system is direct. The LDV scanning head is at coordinate $\mathbf{x}_{L}(-111.3 \mathrm{~cm}, 0,0)$. A reference microphone, denoted Mic \#1, is placed above the spark source at coordinates $(0,0,11.5 \mathrm{~cm})$ (green support in Fig. 2). An additional control microphone, denoted Mic \#2, is placed at coordinates $(0,-71 \mathrm{~cm}, 0)$ (red support in Fig. 2).

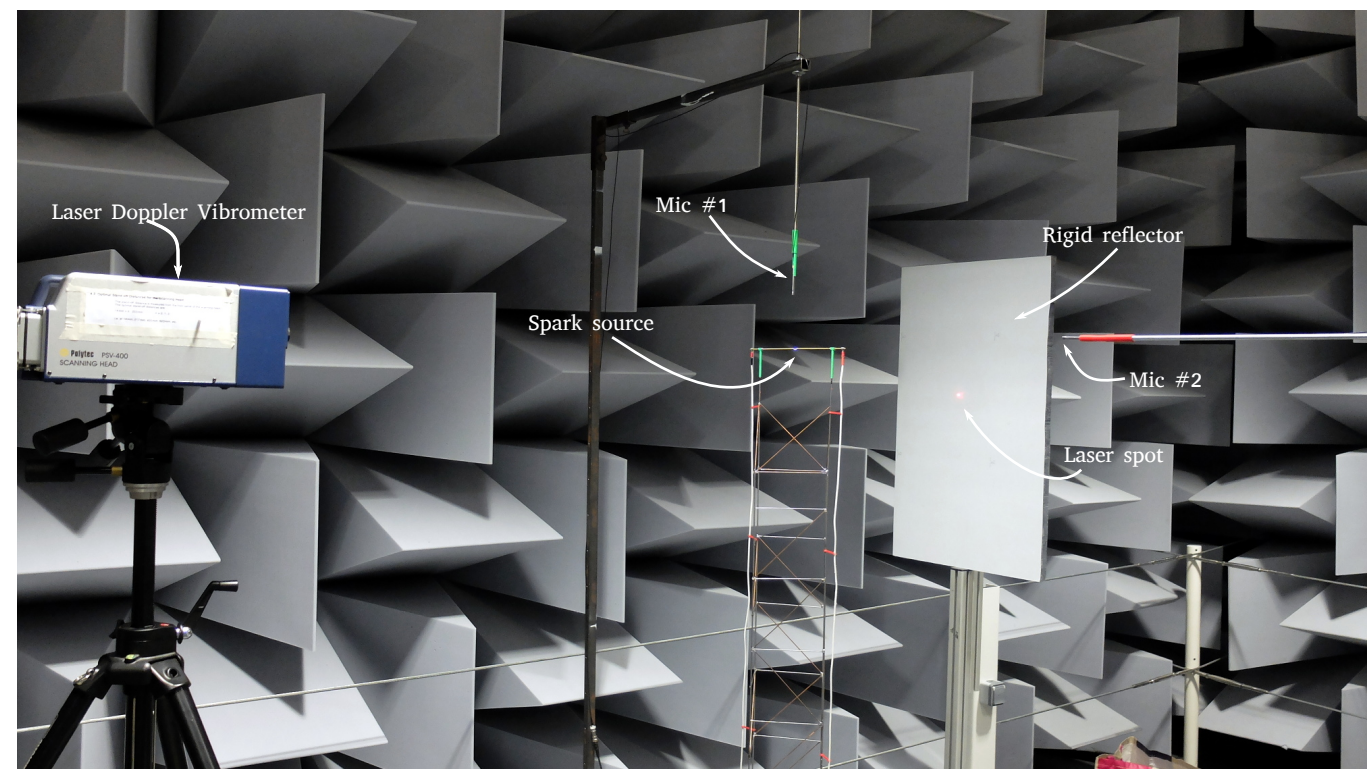

Figure 2: Layout of the experiment: A LDV (left on the picture) emits a laser beam which crosses the sound pressure field and reflects back on the rigid reflector (right of the picture). The acoustic field is radiated by a spark source emitted between two electrodes (middle of the picture). Two quarter-inch microphones are placed at coordinates Mic \# 1: $(0,0,11.5 \mathrm{~cm})$ and Mic \# 2: $(0,-71 \mathrm{~cm}, 0)$, corresponding to green and red support respectively.

Examples of waveforms measured by microphones 1 and 2 are shown in Fig. 4 in black circles. For these measurements, the sampling frequency is $f_{s}=102.4 \mathrm{kHz}$. As a consequence, the acoustic signal of the pulse is strongly lowpass filtered by the anti-aliasing device of the Digital Acquisition System. In addition, the response of microphones Mic \#1 and Mic \#2 filters as well the waveforms. As a consequence, the pulse waveforms on acquired 


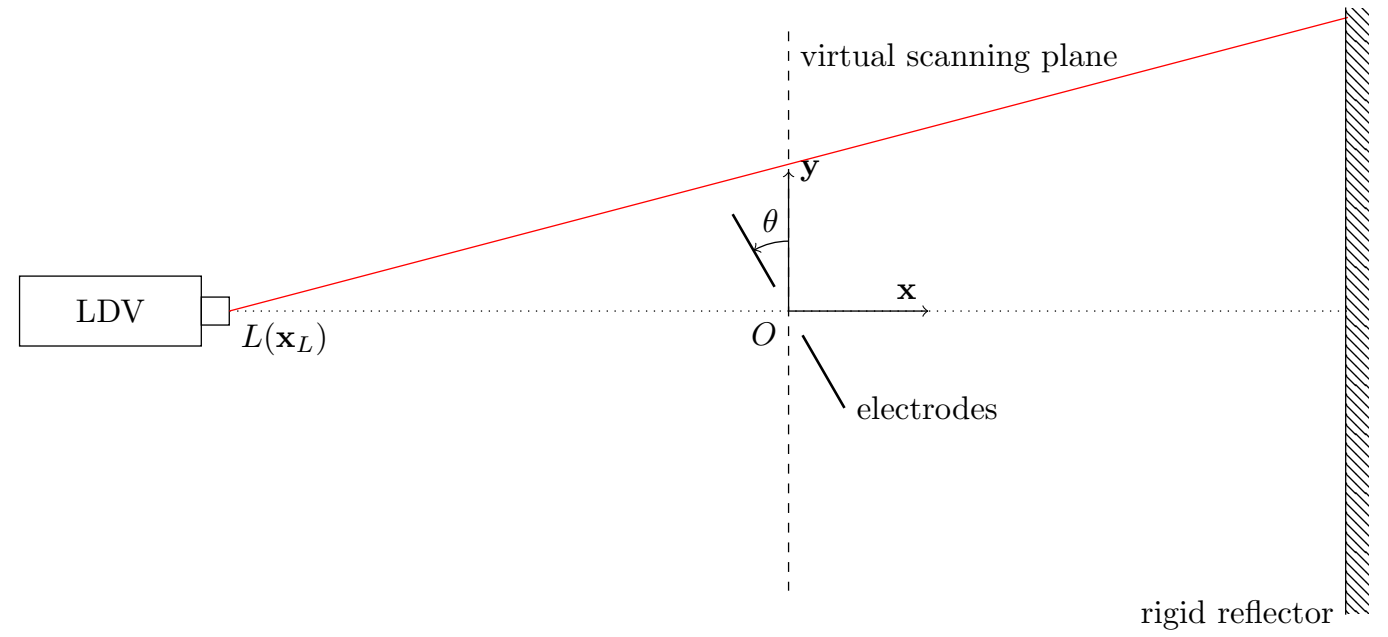

Figure 3: Geometrical layout (top view). The measurement points are defined in the virtual scanning plan $(O y z)$. The electrodes are oriented such that their axis is at an angle $\theta$ from $y$-axis.

signals are not identical to the pressure waveforms, and the analysis of the temporal parameters, such as the rise time, the amplitude or the duration, is compromised $[23,14]$. However, the present study focuses on the "low" frequency characteristics of the source $(<40 \mathrm{kHz})$ which are not affected by these filtering effects. Note that for more readability purposes, the solid line signals in Fig. 4 are oversampled by a factor 10 .

\subsection{Measurement points}

Measurement points are set in the plane $(O y z)$. They are organized following a rectangular mesh of dimensions $\left(L_{y} \simeq 20 \mathrm{~cm} \times L_{z} \simeq 23 \mathrm{~cm}\right)$. The $y$ and $z$ distances between scan points are identically set to $\Delta_{P}=6.1 \mathrm{~mm}$. In this situation, there are $M=1286$ measurement points per spark source orientation. In the reported cases, measurements are operated for 10 different spark source orientations $\theta$ from $\theta_{\min }=0^{\circ}$ to $\theta_{\max }=-90^{\circ}$, with steps of $10^{\circ}$. Note that the scanning grid is the same for the different source orientations, as shown in Fig. 3 .

\subsection{Measurement procedure and parameters}

For each source orientation $\theta$, the LDV scans the 1286-point grid. The acquisition is triggered by a threshold detection on the reference microphone signal (Mic \# 1). More precisely, it is triggered a little before the shock wave 


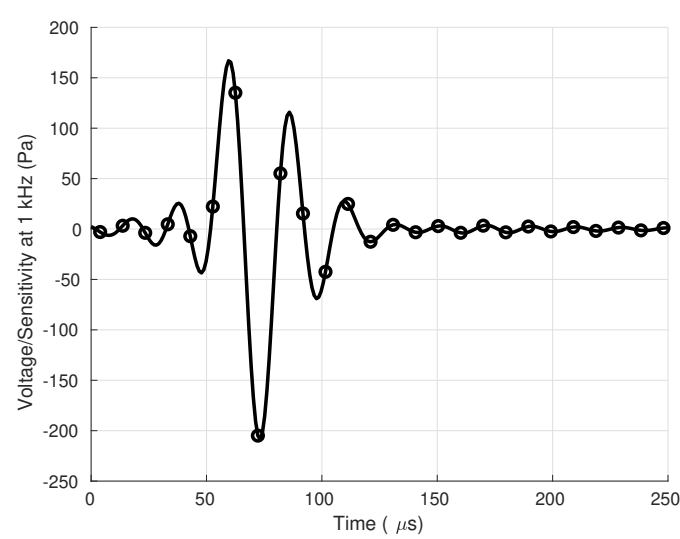

(a)

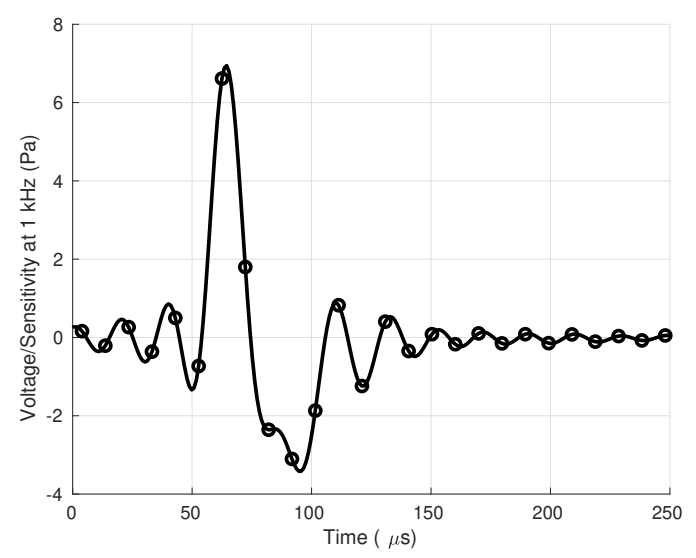

(b)

Figure 4: Example of waveform measured at Mic \#1 $(0,0,11.5 \mathrm{~cm})$ and Mic \#2 $(0$, $-71 \mathrm{~cm}, 0)$ (a) Mic \#1 signal. (b) Mic \# 2 signal. The time origin is set arbitrarily. The acquisition sampling frequency is $f_{s}=102.4 \mathrm{kHz}$. The original samples are plotted as black circles. For better readability, the waveforms are also plotted in solid lines after being oversampled by a factor of 10 .

arrives on the reference microphone, allowing the measurement of points closer to the origin. Acquisition duration is fixed at $625 \mu \mathrm{s}$. This short duration makes it possible to be freed from acoustic reflections, in particular that on the rigid reflector. At the same time, it is long enough to measure the furthest points in the scan grid. Rectangular and Hanning windows are operated on the optical and microphone signals respectively. Sampling frequency is set to $f_{s}=102.4 \mathrm{kHz}$. The frequency range is $[1.6 \mathrm{kHz}, 40 \mathrm{kHz}]$ with a step of $\Delta f=1600 \mathrm{~Hz}$.

The spark source generates a spark approximately every 0.1 s. For each measurement point, auto and cross spectra of the optical and microphone signals are averaged over 10 acquisitions. The LDV apparent velocity at a point of index $m$ is computed from the following formula:

$$
v_{m}=\frac{S_{v_{m}, p_{1, m}}}{S_{p_{1, m}, p_{1, m}}} \sqrt{\left\langle S_{p_{1, m}, p_{1, m}}\right\rangle_{M}} .
$$

In Eq. (17), $S_{v_{m} p_{1, m}}$ is the cross-spectrum between the LDV optical and reference microphone (Mic \#1) signals for measurement of index $m$. $S_{p_{1, m}, p_{1, m}}$ is the auto-spectrum of reference microphone signal (Mic \#1) for the measurement $m$. The operator $\langle\cdot\rangle_{M}$ denotes an average over $M$ measurements. 
Using an average value helps reducing the estimation error on the spectrum $v_{m}[24]$.

\section{Equivalent source method parameters}

\subsection{Equivalent sources layout}

The point sources are distributed around the spark electrodes axis, following a parallelepipedic layout. In the reported case, the distance along $x, y$ and $z$ axis between the equivalent point sources is identical and fixed at $\Delta=1.5 \mathrm{~mm}$. This results in a maximum reproducible frequency of $f_{\max }=\frac{c}{2 \Delta} \simeq 113 \mathrm{kHz}$ [25]. The parallelepiped is centered at the origin and its dimensions are $\left(L_{x} \times L_{y} \times L_{z}\right)=(2 \mathrm{~cm} \times 4 \mathrm{~cm} \times 2 \mathrm{~cm})$. In this situation, there are $N=5292$ point sources. For the acousto-optic interaction described in Sec. 2.1, the distance between the laser beam and an equivalent point source is denoted $a$, and its expression is detailed in Appendix C.

In this situation, the minimum and maximum distances $a$ are $a_{\min }=$ $0.02 \mathrm{~cm}$ and $a_{\max }=17.6 \mathrm{~cm}$.

\subsection{Physical parameters estimation}

The physical parameters $n_{0}, \gamma, p_{0}$ and $c$ in Eq. 12 depend on the environmental conditions and should be calculated accordingly. In the reported case, the static pressure $p_{0}$, temperature, and humidity were measured for each set of acquisition. From these measurements, the sound speed $c$ and the specific heats ratio $\gamma$ were calculated for each set of acquisition according to [26]. The refractive index is taken constant with $n_{0}=1.0002772$.

\subsection{Measurement points selection}

Before solving the inverse problem described in Sec. 3, a sub-set of optical measurements $\mathbf{v}$ are selected with two criteria:

Coherence. The first criterion is based on the coherence between the reference microphone and LDV optical signals [27]. At low frequencies, the spark source radiates little energy. This is due to the spark source energy spectral density nature [28] and it is related to the electrode gap width [29]. Moreover, the velocity output of the LDV is proportional to the time derivative of the acoustic pressure (Eq. (5)). In the frequency domain, this is traduced by a factor $i \omega$ which is low at low frequencies. Consequently, the signal to noise ratio between reference microphone and LDV optical signal is poor and 
the coherence as well. Besides, for some laser incidences and scan points, the laser path can meet an obstacle such as the electrodes support. The coherence criterion is also weak in these situation.

In summary, the selection based on coherence criterion ensures that measurements with low signal-to-noise ratio or wrong laser paths are discarded for the inverse problem. In the reported case, only the measurements with a coherence above 0.8 are retained.

Minimum radius. The second selection criterion is based on the radius between measurement points and origin. In the reported case, only the points at a minimum distance of $1.5 \mathrm{~cm}$ from origin are retained. This ensures that no measurement points are located inside the equivalent source volume and helps with the condition number of the matrix $\mathbf{G}$. In fact, equivalent sources close to measurement points bring low distances a (See Appendix C), leading to high amplitude terms in the matrix $\mathbf{G}$. Moreover, in the near field of the spark source, the non-linear propagation effects are predominant [30] and could disturb the linear equivalent source model. This topic is left for further investigations.

After applying the sub-set selection on the LDV signals, the number of measurement points (i.e. the line number of matrix $\mathbf{G}$ ) is noted $\tilde{M}$.

\subsubsection{Beamforming regularization}

The weighting matrix $\mathbf{W}$ of Eq. (16), is chosen from a delay-and-sum beamforming solution [20]. Thus, $\mathbf{W}$ is a diagonal matrix with its diagonal term $W_{n, n}$ given by:

$$
W_{n, n}=\frac{\left|\mathbf{G}_{n}^{H} v\right|^{2}}{\left\|\mathbf{G}_{n}\right\|^{2}\|\mathbf{v}\|^{2}} .
$$

In Eq. (18), $\mathbf{G}_{n}$ is the $n$-th column of the matrix $\mathbf{G}$ [20]. Finally, the diagonal term of $\mathbf{W}, W_{n, n}$, is a value between 0 and 1 which weights the equivalent source $n$ for the inversion. In the reported cases, all the values such as $W_{n, n}<0.02$ are set to 0 , and the corresponding equivalent sources are discarded for the inversion. After this selection, the number of equivalent sources (i.e. the column number of matrix $\mathbf{G}$ ) is $\tilde{N}$.

\subsubsection{Additional Truncated Singular Value Decomposition}

An additional Truncated Singular Value Decomposition (TSVD) [31] is applied on the matrix $\mathbf{G}^{(\tilde{M} \times \tilde{N})}$ after beamforming regularization. Only the $s$ 
highest singular values are kept with:

$$
s=\left\lceil\max \left(s_{\min },\left(\frac{2}{\lambda}\right)^{3} L_{x} L_{y} L_{z}\right)\right\rceil,
$$

Where $[\cdot]$ represents the rounding up to the nearest integer, $s_{\min }=10$ in the reported case and $\lambda$ is the wavelength. The term $(2 / \lambda)^{3} L_{x} L_{y} L_{z}$ is a criterion which gives the minimum required number of points in the equivalent source volume to avoid spatial aliasing. Therefore, this approach regularizes the matrix to be inverted from a physical criterion. For instance, at low frequencies, the spatial resolution of the equivalent sources layout is unnecessarily dense. It brings many singular values which are discarded with this approach.

\subsection{Results}

This section presents several results for the acoustic pulse equivalent source model.

\subsubsection{Equivalent sources strengths}

The normalized equivalent sources strengths $\left|q_{n}\right|^{2} /\left|q_{n, \max }\right|^{2}$, are displayed at several frequencies in Fig. 5. Each equivalent source is represented as a dot whose size and color is proportional to its strength. The two electrodes are represented as thick lines.

In order to evaluate the reconstruction quality at the scan points, a global coherence criterion, ranging from 0 to 1 , is defined as:

$$
C=\frac{\left|\mathbf{v}^{H} \hat{\mathbf{v}}\right|^{2}}{\|\mathbf{v}\|^{2}\|\hat{\mathbf{v}}\|^{2}}
$$

As well, a degree of over-determination for the inversion is given by:

$$
S=\frac{\tilde{M}}{\tilde{N}} .
$$

These two criteria are displayed for each frequency in Fig. 5.

One observes that the highest equivalent source strengths are gathered in the vicinity of the gap in between the electrodes. This is in accordance with

the spark source spatial location. As the frequency increases, the number of predominant equivalent sources decreases and a line-source shape appears. However, with a closer look, one observes that equivalent sources are located 


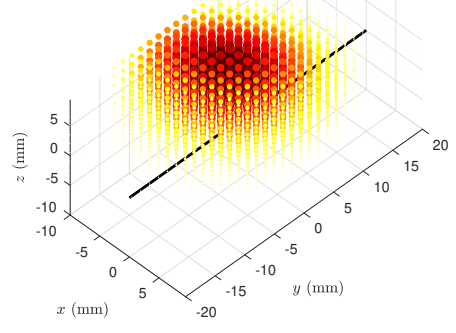

(a)

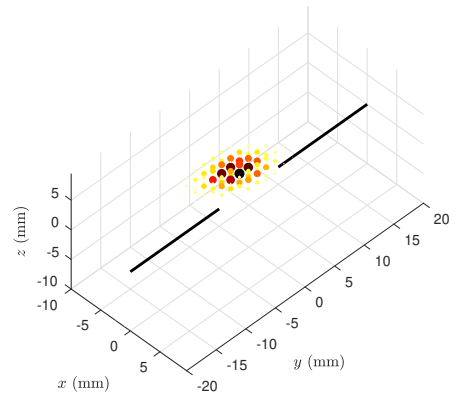

(d)

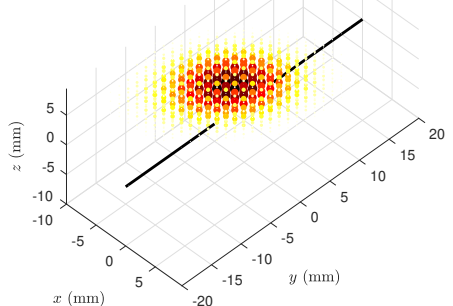

(b)

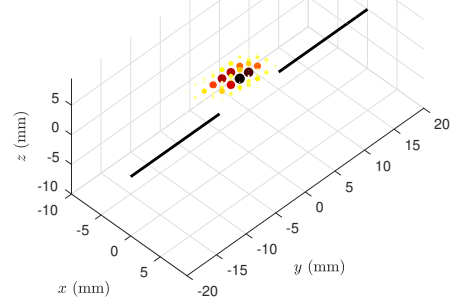

(e)

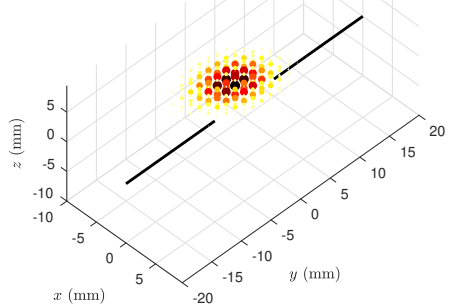

(c)

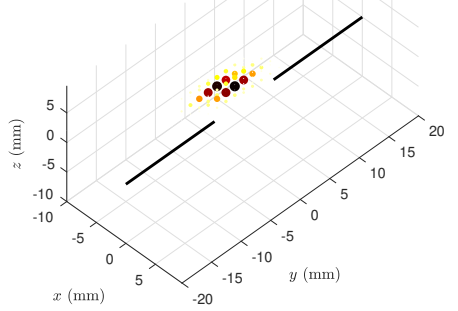

(f)

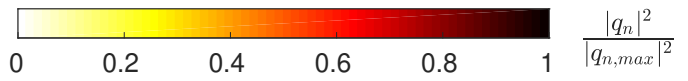

Figure 5: Normalized equivalent sources strengths at several frequencies. The electrodes are represented as black thick lines. The size and color of the sources are proportional to their strength. (a) $f=6400 \mathrm{~Hz}, C=0.97052, S=2.2355$. (b) $f=12800 \mathrm{~Hz}$, $C=0.95703, S=2.3427$. (c) $f=19200 \mathrm{~Hz}, C=0.93532, S=2.3745$. (d) $f=25600 \mathrm{~Hz}$, $C=0.90293, S=2.3998$. (e) $f=32000 \mathrm{~Hz}, C=0.86254, S=1.9696$. (f) $f=38400 \mathrm{~Hz}$, $C=0.81213, S=1.3220$. 
a few millimeters next to the electrode axis. This can be explained by the positioning precision of the experimental layout. As a matter of fact, there are slight misalignment between the LDV, the spark source and the equivalent sources respective coordinate systems. Consequently, the distances $a$ (Eq. (C.4)) can reflect these misalignments and the inverse problem solution as well. In addition, the scans orientations into consideration and their number can influence the reconstruction results. This topic requires further investigations. Regarding the reconstruction quality highlighted by the criterion $C$, one observes that it is ranging from 0.97 at low frequencies $(6400 \mathrm{~Hz})$ to 0.81 at high frequencies $(38.4 \mathrm{kHz})$.

\subsubsection{Reconstructed pressure}

From the equivalent monopole source strengths, the pressure at a point $\mathbf{x}$ can be synthesized with a free field Green function:

$$
p(\mathbf{x}, \omega)=q(\omega) \frac{\mathrm{e}^{-\mathrm{i} \frac{\omega}{c}\left\|x-x_{s}\right\|}}{4 \pi\left\|x-x_{s}\right\|}
$$

The sound pressure levels measured by the microphones Mic \#1 are Mic \#2 and shown on Fig. $6 \mathrm{a}$ and $6 \mathrm{~b}$ respectively. The dashed line curves are the sound pressure levels including free field normal incidence corrections. The correction coefficients are taken from the B\&K(C) datasheets. The solid line curves are the sound pressure levels reconstructed with the equivalent source. From these figures, one notices that the equivalent sources reconstruct accurately the sound pressure level at both locations within the range of $2 \mathrm{~dB}$ for the considered frequencies. However, for the Mic \#2 reconstructed pressure, there are ripples from $12 \mathrm{kHz}$ to $20 \mathrm{kHz}$. By looking at the equivalent sources layout (Fig 5) and their directivity (Fig. 7) at these frequencies, one observes that they are rotated around the vertical axis with respect to the electrode axis. Thus, the pressure reconstructed at the Mic \# 2 is slightly different from the situation where the equivalent sources would be aligned in the electrode axis.

\subsubsection{Equivalent sources directivity}

The normalized directivity at $1 \mathrm{~m}$ of the equivalent sources is shown in Fig. 7 at several frequencies: the equivalent source pressure, Eq. 22, is reconstructed at a radial distance of $1 \mathrm{~m}$ and various angles. Absolute value of this pressure is normalized by the maximum absolute value on the $4 \pi$ steradian angle. One can see that the equivalent source directivities range 


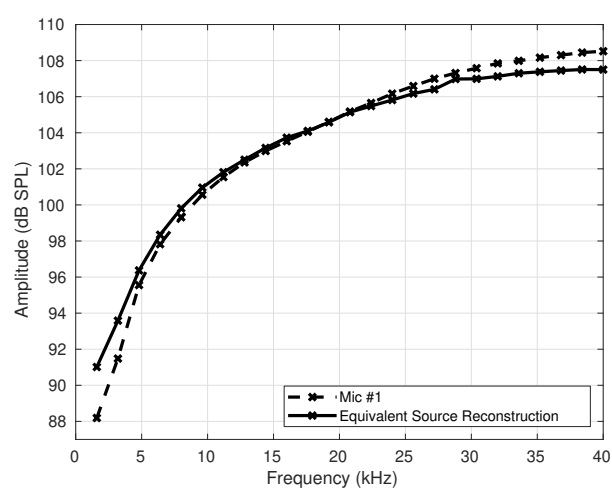

(a)

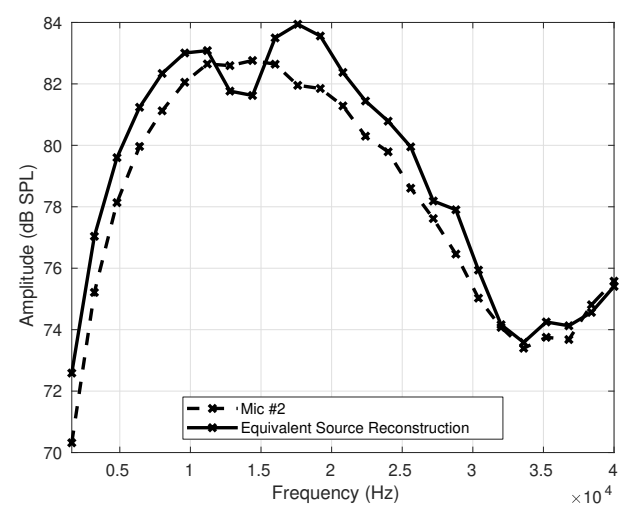

(b)

Figure 6: Measured and reconstructed sound pressure level in dB SPL (reference $20 \times$ $\left.10^{-6} \mathrm{~Pa}\right)$ : (a) Microphone \#1 $(0,0,11.5 \mathrm{~cm})$. (b) Microphone \#2 $(0,-71 \mathrm{~cm}, 0)$. Dashed line curves: measured pressure including free field normal incidence corrections. Solid line curves: equivalent source reconstruction.

from a quasi-omnidirectionnnal source at low frequencies to a line source directivity at higher frequencies. This is in accordance with the literature a spark source directivity generated between two electrodes [29]. However, at mid frequencies, from $12 \mathrm{kHz}$ to $20 \mathrm{kHz}$, the source orientation is shifted from the electrode axis. This is confirmed by the observation of equivalent source spatial distribution at these frequencies in Fig. 5.

\subsection{Discussion}

The results presented in an experimental context for an acoustic pulse show an equivalent sources model from acousto-optic measurements. The inverse problem solutions is regularized with a beamforming solution and an additional TSVD. The equivalent sources are found in the vicinity of the spark source gap. The direct problem reconstruction give high coherence values in comparison with the measurements. The reconstructed pressures at control microphones are accurate and the directivity of the equivalent source is in accordance with a line-source shape. However, several factors influence the results and are discussed here.

Positioning accuracy. In an experimental context, the positioning accuracy is limited and influences directly the localization/directivity of the equivalent sources, especially at high frequencies. In fact, for the experimental layout 


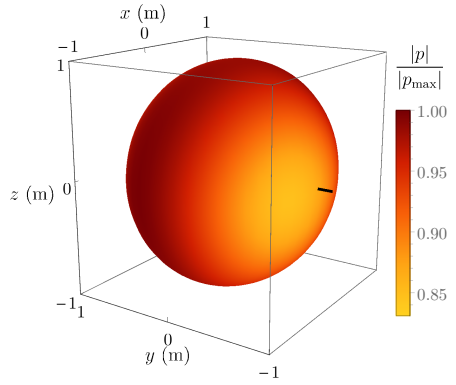

(a)

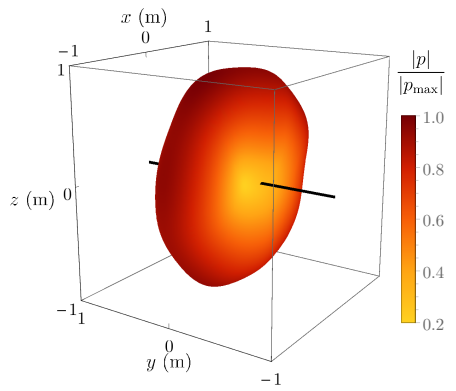

(d)

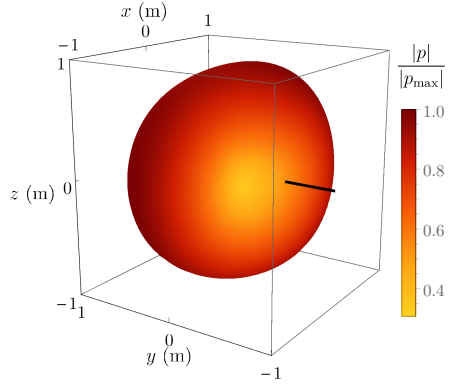

(b)

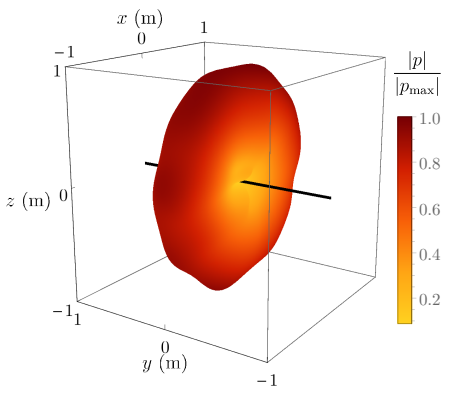

(e)

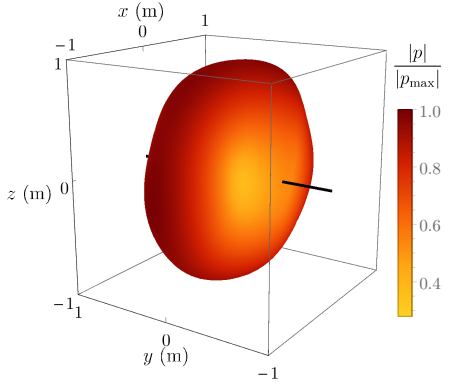

(c)

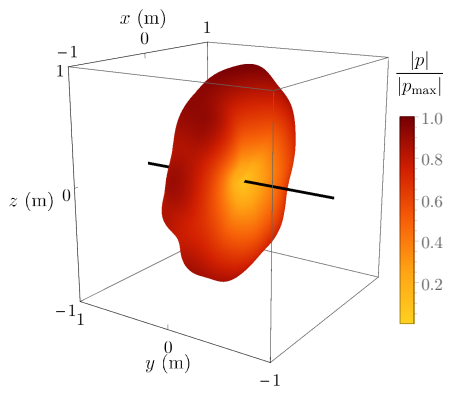

(f)

Figure 7: Equivalent source 3D directivity at $1 \mathrm{~m}$ for several frequencies. Amplitude and color are normalized by the maximum value in the $4 \pi$ steradian angle. (a) $f=6400 \mathrm{~Hz}$. (b) $f=12800 \mathrm{~Hz}$. (a) $f=19200 \mathrm{~Hz}$. (b) $f=25600 \mathrm{~Hz}$. (a) $f=32000 \mathrm{~Hz}$. (b) $f=38400 \mathrm{~Hz}$. 
described in the paper, the positioning accuracy is around the half-centimeter range. Moreover, the spark source rotation axis is not exactly coincident with the equivalent sources rotation axis. Consequently, when computing the distances $a$ for a theoretical configuration, a bias is introduced and compromises the results, as one can observes for the equivalent source location and orientation. The study of the positioning accuracy for the robustness of the proposed method is a topic for further investigations.

LDV laser beam focusing. Using a scanning LDV offers the possibility of measuring numerous points in an automated way. As the device is not moving during the scan, it uses lenses and mirrors to focus the laser on each measurement point location. As a result, the laser has a varying diameter along the integration path. When this diameter is of the same order of magnitude as the acoustic wavelength, an averaging on the laser cross section is done [32, p.45-46]. To minimize this effect, it is advised to focus the laser beam where the acousto-optic effect is pre-dominant, i.e. in the vicinity of the spark source for the current experiment. However, by doing so, the signal-to-noise ratio decreases as there will be less light reflected on the rigid surface. In the current experiment, the laser is focused on the rigid reflector in Fig. 2.

Signal to noise ratio. Using an LDV for measuring the acousto-optic effect is a misuse of such a device. In fact, a LDV is sensitive to the mechanical vibrations of a moving surface (first right-hand term in Eq. (3)). As the acousto-optic effect (second right-hand term in Eq. (3)) is weak in comparison with the mechanical vibration, a high signal-to-noise ratio is needed to observe it with an LDV. This implies several limitations for the acoustic sources in an experimental context. A first solution is to drive the acoustic source in harmonic mode with a single frequency, to increase the signal-tonoise ratio [13]. In the present case and transient spark source is used, and a limited duration acquisition is done to maximize the signal-to-noise ratio. However, the acousto-optic effect decreases rapidly as the laser distance to the source increases. This limits the measurement to near field of the spark source. A solution would be to increase the electrode gap, but this will increases the non-linear propagations effects as mentioned in the following paragraph. An other lead would be to use more sensible interferometers, such as Mach-Zender interferometers [14].

Spark source propagation. The spark source presents several advantages in an experimental context. It is a transient source which allows the characteri- 
zation in a non-anechoic environment. The finite duration acquisition allows to discard the wave reflections on the rooms walls, LDV scanning head of rigid reflector. Moreover, it is an transparent source, i.e. the laser beam has no obstacle on its path, except the electrodes and their support in the case of and electric spark source. This allows to measure the acousto-optic effect with various incidence and enhance its characterization. However, a spark source typically propagates with nonlinearities [30]. These effects are predominant in the near field of the spark source gap and increase as the electrode gap increases [29]. Besides, in the proposed method, a linear superposition of linear monopole sources is used to construct and equivalent sources model. Consequently, the equivalent source model is valid in a leastsquare sense but it may not be the most physically valid solution. To improve this point, it would be possible to describe the acouto-optic effect for an elementary point source propagating with nonlinearites. This topic is left for further investigations.

\section{Conclusion and Future Works}

This paper presented a method for acoustic sources characterization using optical measurements: the acousto-optic effect is measured with a LDV at various distance and incidence from the sources. From these measurements, an inverse problem is solved in order to construct and equivalent monopole sources model. The model uses an acousto-optical Green function.

Effects of finite length laser beam and acquisition duration were studied. The method was validated in an experimental context for an acoustic pulse generated with a spark source. Results showed good agreement for the equivalent sources model in term of spatial location, reconstructed pressures and directivity.

The main drawbacks of the experimental method were highlighted. In particular, it would be advised to use more sensible optical interferometers.

Future works include the comparison of the proposed method with acoustooptic tomography [13] and studies of the method robustness to positioning accuracy, number and orientation of measurements, and nonlinear effects. For this latter point, a nonlinear propagation code for spark source will be used [33]. 


\section{Acknowledgments}

This work was supported by the Labex CeLyA of Université de Lyon, operated by the French National Research Agency (ANR-10-LABX-0060/ ANR-11-IDEX-0007).

\section{Appendix A. Computation of integral $\boldsymbol{I}$ in Eq. (8)}

The integral $I(t)$ of Eq. (8) is recalled here for readability:

$$
I(t)=\int_{-\alpha}^{\alpha} \frac{\delta\left(t-\frac{\sqrt{a^{2}+x^{2}}}{c}\right)}{\sqrt{a^{2}+x^{2}}} \mathrm{~d} x .
$$

The function under the integral in $I$ is pair with respect to variable $x$, thus:

$$
I(t)=2 \int_{0}^{\alpha} \frac{\delta\left(t-\frac{\sqrt{a^{2}+x^{2}}}{c}\right)}{\sqrt{a^{2}+x^{2}}} \mathrm{~d} x .
$$

In order to compute integral $I$ in Eq. (8), two situations are considered with respect to variable $t$.

. The first situation is when $\left.t \notin] \frac{a}{c}, \frac{\sqrt{a^{2}+\alpha^{2}}}{c}\right]$, that is to say when the spatial Dirac pulse is outside the laser beam in spatial interval $[-\alpha, \alpha]$. In this situation,

$$
I(t)=0 .
$$

. The second situation is for $\left.t \in] \frac{a}{c}, \frac{\sqrt{a^{2}+\alpha^{2}}}{c}\right]$, that is to say, when the spatial Dirac pulse is crossed by the laser beam in spatial interval $[-\alpha, \alpha]$.

Let $u \in\left[t-\frac{\sqrt{a^{2}+\alpha^{2}}}{c}, t-\frac{a}{c}\right]$ be the variable such that:

$$
u=t-\frac{\sqrt{a^{2}+x^{2}}}{c} .
$$

From the condition on $t, u \in]-\beta, \beta]$, with $\beta=\frac{\sqrt{a^{2}+\alpha^{2}}-a}{c}$, and from Eq.(A.4), one has:

$$
x^{2}=c^{2}(t-u)^{2}-a^{2} .
$$

As $\left.\left.c^{2}(t-u)^{2} \in\right] a^{2}, a^{2}+\alpha^{2}\right]$ :

$$
x=\sqrt{c^{2}(t-u)^{2}-a^{2}},
$$




$$
\begin{gathered}
\mathrm{d} u=\frac{-x}{c \sqrt{a^{2}+x^{2}}} \mathrm{~d} x \\
\mathrm{~d} u=\frac{-\sqrt{c^{2}(t-u)^{2}-a^{2}}}{c \sqrt{a^{2}+x^{2}}} \mathrm{~d} x, \\
\frac{\mathrm{d} x}{\sqrt{a^{2}+x^{2}}}=\frac{-c \mathrm{~d} u}{\sqrt{c^{2}(t-u)^{2}-a^{2}}} .
\end{gathered}
$$

By doing the variable change with $u$, one has:

$$
I(t)=-2 \int_{-\beta}^{\beta} \frac{-c \delta(u)}{\sqrt{c^{2}(t-u)^{2}-a^{2}}} \mathrm{~d} u,
$$

Finally:

$$
I(t)=\frac{2 c}{a} \frac{1}{\sqrt{\frac{c^{2}}{a^{2}} t^{2}-1}} .
$$

The two aforementioned situations can be grouped with an indicator function $\mathbb{1}_{\left.] \frac{a}{c}, \frac{\sqrt{a^{2}+\alpha^{2}}}{c}\right]}(t)$ such that:

$$
\mathbb{1}_{] \frac{a}{c}, \frac{\sqrt{a^{2}+\alpha^{2}}}{c}\right]}(t)= \begin{cases}1 & \left.t \in] \frac{a}{c}, \frac{\sqrt{a^{2}+\alpha^{2}}}{c}\right] \\ 0 & \left.t \notin] \frac{a}{c}, \frac{\sqrt{a^{2}+\alpha^{2}}}{c}\right]\end{cases}
$$

Finally:

$$
I(t)=\mathbb{1}_{] \frac{a}{c}, \frac{\sqrt{a^{2}+\alpha^{2}}}{c}\right]} \frac{2 c}{a} \frac{1}{\sqrt{\frac{c^{2}}{a^{2}} t^{2}-1}} .
$$

In order to work in the frequency domain, the Fourier transform of $I(t)$ is computed as follows:

$$
\begin{gathered}
I(\omega)=\int_{-\infty}^{+\infty} I(t) \mathrm{e}^{-\mathrm{i} \omega t} d t \\
I(\omega)=\int_{\frac{a}{c}}^{\frac{\sqrt{a^{2}+\alpha^{2}}}{c}} \frac{2 c}{a} \frac{1}{\sqrt{\frac{c^{2}}{a^{2}} t^{2}-1}} \mathrm{e}^{-\mathrm{i} \omega t} \mathrm{~d} t
\end{gathered}
$$


With variable change $u=\frac{c t}{a}, d u=\frac{c}{a} \mathrm{~d} t$, one has:

$$
I(\omega)=2 \int_{1}^{\frac{\sqrt{a^{2}+\alpha^{2}}}{a}} \frac{1}{\sqrt{u^{2}-1}} \mathrm{e}^{-\mathrm{i} \omega \frac{a}{c} u} \mathrm{~d} u
$$

One can split the previous integral in two parts:

$$
I(\omega)=2 \int_{1}^{+\infty} \frac{1}{\sqrt{u^{2}-1}} \mathrm{e}^{-\mathrm{i} \omega \frac{a}{c} u} \mathrm{~d} u-2 \int_{\frac{\sqrt{a^{2}+\alpha^{2}}}{a}}^{+\infty} \frac{1}{\sqrt{u^{2}-1}} \mathrm{e}^{-\mathrm{i} \omega \frac{a}{c} u} \mathrm{~d} u
$$

The first term of the right-hand side in $\mathrm{Eq}$ (A.17) is an integral form of a modified Bessel functions of second kind of degree 0, $K_{0}$ [34, Eq. 9.6.23]. It corresponds to the situation where the laser beam is of infinite length and the recording duration is infinitely long. Thus,

$$
I(\omega)=2 K_{0}(\mathrm{i} \omega a / c)-2 \int_{\frac{\sqrt{a^{2}+\alpha^{2}}}{a}}^{+\infty} \frac{1}{\sqrt{u^{2}-1}} \mathrm{e}^{-\mathrm{i} \omega \frac{a}{c} u} \mathrm{~d} u
$$

\section{Appendix B. Study of the residual in Eq. (A.18)}

The integral $I(\omega)$ can be approximated by $K_{0}(i \omega a / c)$ if the second term of the right-hand side in Eq. (A.18) is negligible when compared to $K_{0}(i \omega a / c)$. In order to study this residual, one expresses the following term:

$$
R(\lambda, a, \alpha)=\frac{2 \int_{\sqrt{a^{2}+\alpha^{2}} / a}^{+\infty} \frac{e^{-\mathrm{i} 2 \pi \frac{a}{\lambda} u}}{\sqrt{u^{2}-1}} \mathrm{~d} u}{I(\lambda, a, \alpha)},
$$

where $\lambda=c / f$ is the acoustical wavelength. The magnitude $R$ is shown in Fig. B.8. One observes that it decreases as the ratio $a / \lambda$ decreases and the ratio $\alpha / \lambda$ increases. Physically, this means that the error of approximation decreases when the laser length is big in comparison to the acoustical wavelength and when the distance $a$ is small in comparison to the acoustical wavelength. In other words, a long laser, close to the point source, relatively to the acoustical wavelength, decreases the approximation error. For a practical application where the laser length is not changing but the distance to the source is, $R$ can be plotted versus frequency, as shown in Fig. B.9. This situation corresponds to the experiment described in Sec. 4. In Fig. B.9, the laser-length is set to $1 \mathrm{~m}$ and the sound speed to $c=340 \mathrm{~m} / \mathrm{s}$. In 


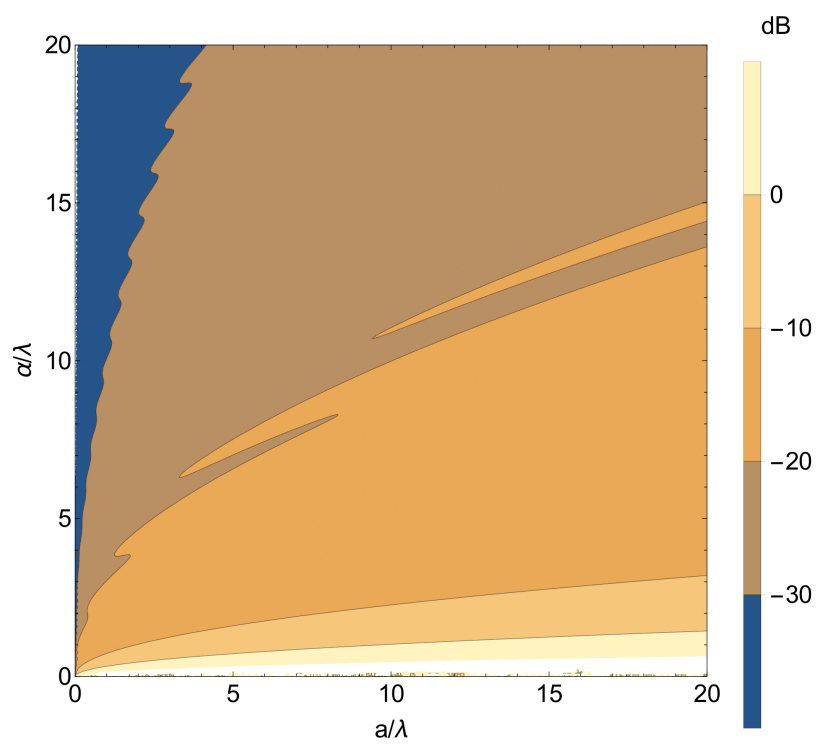

Figure B.8: Contour plots for the magnitude of term $R$ (Eq. (B.1)) versus the ratios $a / \lambda$ and $\alpha / \lambda$.

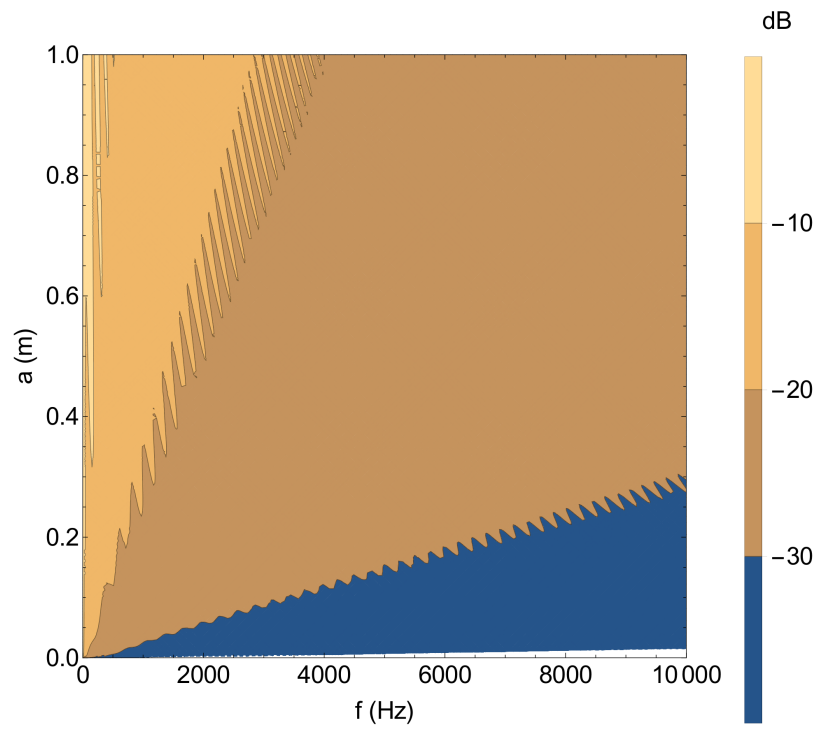

Figure B.9: Contour plots for the magnitude of term $R$ (Eq. (B.1)) versus the distance laser-point-source, $a$, and the frequency $f$. In Eq. (B.1), the sound speed is set to $c=$ $340 \mathrm{~m} / \mathrm{s}$ and the laser length to $1 \mathrm{~m}$. 
this case, one observes that the residual $R$ decreases as the frequency and point-source-distance increase. For instance, in the measurement points and equivalent source layouts described in Sec. 4.3 and Sec 5.1 respectively, the distance $a$ spans from $a_{\min }=0.02 \mathrm{~cm}$ to $a_{\max }=17.4 \mathrm{~cm}$, and the minimum measurement frequency is $1600 \mathrm{~Hz}$. In this situation, $R$ is always inferior to $-20 \mathrm{~dB}$.

\section{Appendix C. Analytic expression of function $a$}

Let $S\left(\mathbf{x}_{n}\right)$ be a monopole at spatial location $\mathbf{x}_{n}$. Let $P\left(\mathbf{x}_{m}\right)$ be a LDV measurement point at spatial location $\mathbf{x}_{m}$. Finally, let $L\left(\mathbf{x}_{L}\right)$ be the LDV laser beam origin. By considering the plane containing the points $(S, P, L)$, the geometrical layout is schematized in Fig. C.10:

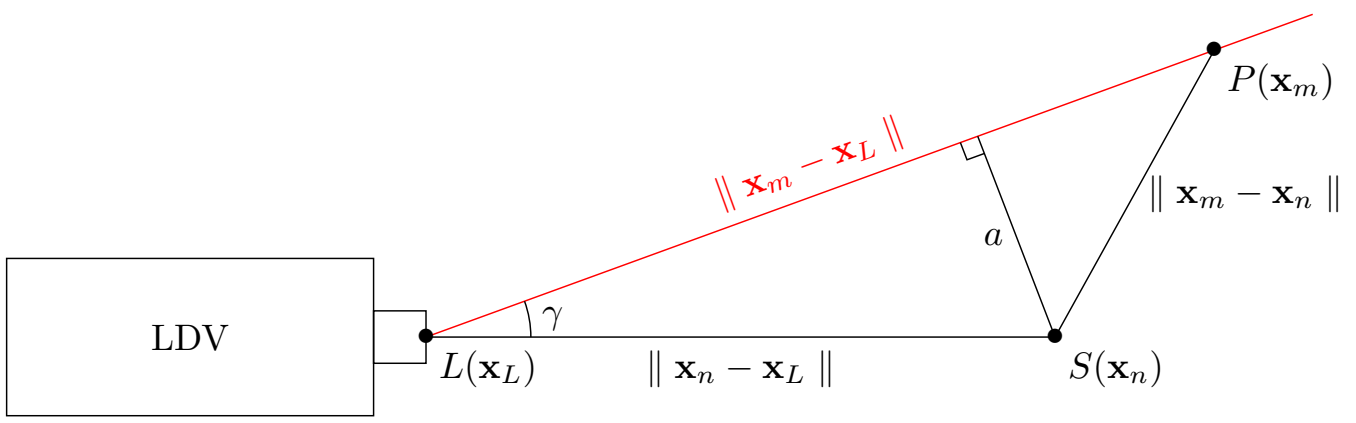

Figure C.10: Geometrical layout in the plane $(S, P, L)$. The laser beam, in red, crosses the scan point $P$ at a distance $a$ from the source $S$.

From law of cosines one has:

$$
\gamma=\arccos \left(\frac{\left\|\mathbf{x}_{n}-\mathbf{x}_{L}\right\|^{2}+\left\|\mathbf{x}_{m}-\mathbf{x}_{L}\right\|^{2}-\left\|\mathbf{x}_{m}-\mathbf{x}_{n}\right\|^{2}}{2\left\|\mathbf{x}_{n}-\mathbf{x}_{L}\right\|\left\|\mathbf{x}_{m}-\mathbf{x}_{L}\right\|}\right)
$$

As well, from Pythagorean theorem, one has:

$$
\sin (\gamma)=\frac{a}{\left\|\mathbf{x}_{n}-\mathbf{x}_{L}\right\|}
$$

Thus,

$a=\left\|\mathbf{x}_{n}-\mathbf{x}_{L}\right\| \sin \left(\arccos \left(\frac{\left\|\mathbf{x}_{n}-\mathbf{x}_{L}\right\|^{2}+\left\|\mathbf{x}_{m}-\mathbf{x}_{L}\right\|^{2}-\left\|\mathbf{x}_{m}-\mathbf{x}_{n}\right\|^{2}}{2\left\|\mathbf{x}_{n}-\mathbf{x}_{L}\right\|\left\|\mathbf{x}_{m}-\mathbf{x}_{L}\right\|}\right)\right)$ 
Finally,

$$
a=\left\|\mathbf{x}_{n}-\mathbf{x}_{L}\right\| \sqrt{1-\left(\frac{\left\|\mathbf{x}_{n}-\mathbf{x}_{L}\right\|^{2}+\left\|\mathbf{x}_{m}-\mathbf{x}_{L}\right\|^{2}-\left\|\mathbf{x}_{m}-\mathbf{x}_{n}\right\|^{2}}{2\left\|\mathbf{x}_{n}-\mathbf{x}_{L}\right\|\left\|\mathbf{x}_{m}-\mathbf{x}_{L}\right\|}\right)^{2}}
$$

\section{References}

[1] J. Billingsley, R. Kinns, The acoustic telescope, Journal of Sound and Vibration 48 (4) (1976) 485-510.

[2] E. G. Williams, Fourier Acoustics: Sound Radiation And Nearfield Acoustical Holography, Academic Press, London, 1999.

[3] G. H. Koopmann, L. Song, J. B. Fahnline, A method for computing acoustic fields based on the principle of wave superposition, The Journal of the Acoustical Society of America 86 (6) (1989) 2433-2438.

[4] P. A. Nelson, S.-H. Yoon, Estimation of acoustic source strength by inverse methods: Part I, conditioning of the inverse problem, Journal of Sound and Vibration 233 (4) (2000) 639-664.

[5] M. Fink, Time reversal of ultrasonic fields. I. Basic principles, IEEE transactions on ultrasonics, ferroelectrics, and frequency control 39 (5) (1992) 555-566.

[6] H. Teutsch, Modal Array Signal Processing: Principles and Applications of Acoustic Wavefield Decomposition, Springer, Heidelberg, 2007.

[7] P. Yuldashev, S. Ollivier, M. Averiyanov, O. Sapozhnikov, V. Khokhlova, P. Blanc-Benon, Nonlinear propagation of sparkgenerated N-waves in air: Modeling and measurements using acoustical and optical methods, The Journal of the Acoustical Society of America 128 (6) (2010) 3321-3333.

[8] A. Torras-Rosell, S. B. Figueroa, An Acousto-Optic Method For FreeField Microphone Calibration, in: ICSV 22, Florence, 2015, pp. 1-8.

[9] C. F. Quate, C. D. W. Wilkinson, D. K. Winslow, Interaction of light and microwave sound, Proceedings of the IEEE 53 (10) (1965) 16041623. 
[10] R. Adler, Interaction between light and sound, IEEE spectrum 4 (5) (1967) 42-54.

[11] T. A. Pitts, J. F. Greenleaf, Three-dimensional optical measurement of instantaneous pressure, The Journal of the Acoustical Society of America 108 (6) (2000) 2873-2883.

[12] A. Torras-Rosell, S. Barrera-Figueroa, F. Jacobsen, An acousto-optic beamformer, The Journal of the Acoustical Society of America 132 (1) (2012) 144-149.

[13] A. Torras-Rosell, S. Barrera-Figueroa, F. Jacobsen, Sound field reconstruction using acousto-optic tomography, The Journal of the Acoustical Society of America 131 (5) (2012) 3786-3793.

[14] P. Yuldashev, M. Karzova, V. Khokhlova, S. Ollivier, P. Blanc-Benon, Mach-Zehnder interferometry method for acoustic shock wave measurements in air and broadband calibration of microphones, The Journal of the Acoustical Society of America 137 (6) (2015) 3314-3324.

[15] L. Zipser, Interferometric visualization of generation and propagation of sound, in: ICA 2004, Vol. 2, Kyoto, 2004, pp. 1351-1354.

[16] L. Zipser, H. Franke, Refracto-Vibrometry for Visualizing Ultrasound in Gases, Fluids and Condensed Matter, in: 2007 IEEE Ultrasonics Symposium, IEEE, New York, 2007, pp. 395-398.

[17] L. Zipser, H. H. Franke, Refracto-vibrometry - a novel method for visualizing sound waves in transparent media, Journal of the Acoustical Society of America 123 (5) (2008) 3314.

[18] L. Song, G. H. Koopmann, J. B. Fahnline, Numerical errors associated with the method of superposition for computing acoustic fields, The Journal of the Acoustical Society of America 89 (6) (1991) 2625-2633.

[19] R. Jeans, I. C. Mathews, The wave superposition method as a robust technique for computing acoustic fields, The Journal of the Acoustical Society of America 92 (2) (1992) 1156-1166.

[20] P.-A. Gauthier, C. Camier, Y. Pasco, A. Berry, E. Chambatte, R. Lapointe, M.-A. Delalay, Beamforming regularization matrix and inverse 
problems applied to sound field measurement and extrapolation using microphone array, Journal of Sound and Vibration 330 (24) (2011) 58525877.

[21] A. N. Tikhonov, V. Y. Arsenin, Methods for Solving Ill-Posed Problems, John Wiley and Sons, Inc, Washington, D. C., 1977.

[22] A. Pereira, J. Antoni, Q. Leclere, Empirical Bayesian regularization of the inverse acoustic problem, Applied Acoustics 97 (2015) 11-29.

[23] W. M. Wright, Propagation in air of $\mathrm{N}$ waves produced by sparks, The Journal of the Acoustical Society of America 73 (6) (1983) 1948-1955.

[24] Q. Leclère, Multi-channel spectral analysis of multi-pass acquisition measurements, Mechanical Systems and Signal Processing 23 (5) (2009) 1415-1422. doi:10.1016/j.ymssp.2008.12.002.

[25] S. Spors, R. Rabenstein, Spatial aliasing artifacts produced by linear and circular loudspeaker arrays used for wave field synthesis, in: Audio Engineering Society Convention 120, AES, Paris, 2006, pp. 1-14.

[26] O. Cramer, The variation of the specific heat ratio and the speed of sound in air with temperature, pressure, humidity, and CO2 concentration, The Journal of the Acoustical Society of America 93 (5) (1993) 2510-2516.

[27] A. V. Oppenheim, R. W. Schafer, Digital Signal Processing, PrenticeHall, Englewood Cliffs, NJ, 1975.

[28] J. S. Lee, H. L. Peterson, C. McCoy Jr, Energy spectral density of the sonic boom, The Journal of the Acoustical Society of America 44 (1) (1968) 299-300.

[29] W. M. Wright, N. W. Medendorp, Acoustic Radiation from a Finite Line Source with N-Wave Excitation, The Journal of the Acoustical Society of America 43 (5) (1968) 966-971.

[30] P. V. Yuldashev, M. V. Averiyanov, V. A. Khokhlova, S. Ollivier, P. Blanc-Benon, Nonlinear spherically divergent shock waves propagating in a relaxing medium, Acoustical Physics 54 (1) (2008) 32-41. 
[31] P. C. Hansen, Rank-Deficient and Discrete Ill-Posed Problems: Numerical Aspects of Linear Inversion, Society For Industrial and Applied Mathematics, Philadelphia, 1998.

[32] A. Torras-Rosell, F. Jacobsen, F. T. Agerkvist, S. Barrera Figueroa, New measurements techniques: Optical methods for characterizing sound fields, Ph.D. thesis, Technical University of Denmark (2014).

[33] T. Lechat, D. Dragna, S. Ollivier, Simulation du champ proche généré par une source impulsionnelle à arc électriques (Simulation of the near field generated by an electric arc pulse source), in: CFA 2018, SFA, Le Havre, 2018, pp. 939-945.

[34] M. Abramowitz, I. A. Stegun, Handbook of Mathematical Functions: With Formulas, Graphs, and Mathematical Tables, 10th Edition, National Bureau of Standards, Washington, D. C., 1972. 